\title{
Research on Abrasive Belt Based Machining Technology for Milling Shaped Aero-Engine Blades
}

\author{
Lei QIU, Xi ZENG, Shiming JI, Dapeng TAN, Man GE, Wenbin QIU
}

\begin{abstract}
This paper proposes an automatic surface treatment method for CNC milled blades. The surface texture characteristics of the blade after milling were analyzed. The contact area between the contact wheel and the blade surface was analyzed theoretically, and then the contact wheel and the blade edge were analyzed. The contact area between the intake side and the exhaust side was theoretically analyzed, and one micro-element in the contact area was selected, and the simulation analysis under multiple sets of parameters was carried out. In the experimental aspect, this paper has assembled a set of relatively reliable experimental systems, and carried out an indepth analysis of a certain area of the engine blade obtained from the experiment to verify the feasibility and reliability of the proposed method.
\end{abstract}

Keywords: abrasive belt; aero-engine blade; aviation manufacturing; removal model; surface processing

\section{INTRODUCTION}

The blades function is to form the continuous changing geometric characteristics of the gas section by cooperating with the engine channel cavity, so that the internal gas will be highly compressed and at high temperature. Meanwhile the gas has high flow speed [1]. Finally, the chemical energy of the gas can be transformed into the propulsion power of the aircraft. So blade is the key part of the turbojet engine, which has a great influence on the performance of the turbojet engine [2-4].

The blades shapes are very complex, they are usually designed to distortedly variable cross-section surface [4, 5], which has an important effect on the efficiency, thrust and the air flow direction. In order to meet the requirements of high performance of the engine, which is represented by safety, reliability and long life requirement, the blades must have accurate cross-section shape and appropriate size, strict surface integrity, and allow blade to have a certain angle of torsion deformation [6, 7]. The factors affecting blade surface quality are variable, such as blade shape, surface roughness, waviness, curvature radius of the inlet and exhaust edges, working edge and the surface smoothness $[8,9]$. It is a key factor in the manufacturing field of aero-engine to make the blade with high geometric precision and good surface quality. However, it is difficult to process the blades. In the course of the development of the aviation industry, the processing technology of the blade has been greatly improved. There are several common machining methods for aero-engine blades, such as electrochemical machining, precision forging, precision casting.

Fig. 1 shows the blades produced by Wuxi Hyatech Technology Co. Ltd and blades produced by Ufa engine production joint factory. Blades from Hyatech were processed with precision forging method, which keeps the continuity of the metal streamline, increases the strength and bearing capacity of the blade, and improves the performance and life of the blade to a great extent [10-12]. Blades from Ufa are processed with precision casting method, which makes the blade have a streamlined outer ring profile and even forms an inner cavity of blade for cooling. Due to the spatial curvature and uneven thickness, the dimensional accuracy control is difficult. The quality of the wax mold directly determines the dimensional accuracy of the final casting $[13,14]$.

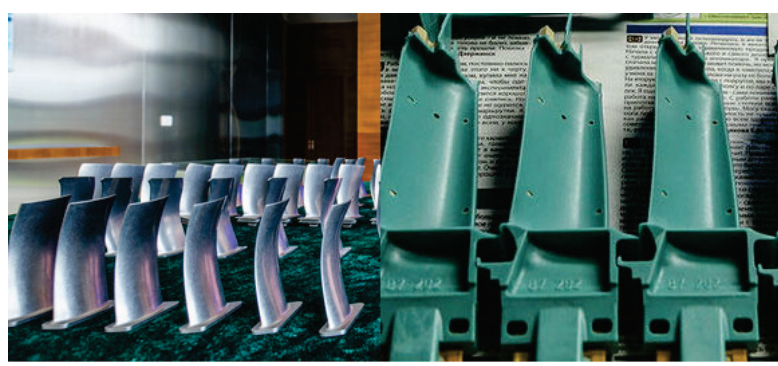

Figure 1 Blades Produced by Wuxi Hyatech Technology Co. Ltd and blades produced by Ufa engine production joint factory

We can get high precision blade finished products by using above methods, but the cost is expensive and the processing term is very long. With the development of numerical control technology, the blade milling technology has been used more and more widely. The most commonly used form of processing is spiral milling, which is very suitable for high quality blade surface machining.

\section{BLADE MILLING AND SURFACE TEXTURE}

As mentioned in the previous articles, the blade NC milling technology cannot only remove most remainder of the blade face and blade bump, but also ensures the blade shape and size fully meet the design requirements [15]. After fine polishing, the blade profile will own good consistency. However, there are great stresses and deformations in the blades processed by the NC milling technology. In some situations the thin blades even can be scrapped due to the great deformation [16].

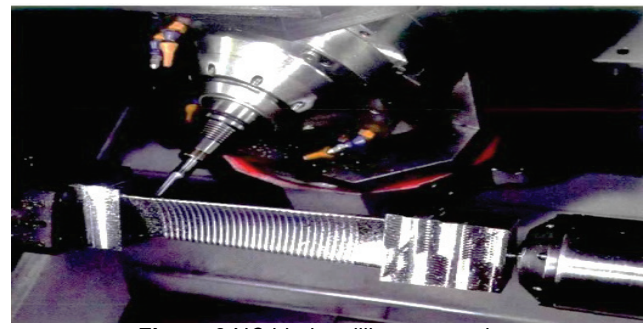

Figure 2 NC blade milling processing 
Machine tool vibration and blade material deformation will have an effect on the formation process of blade surface topography. Therefore, the quality of the blade surface after milling is related to the feed residual height of the tool. Fig. 2 shows the basic situation of blade processing. As we see, there is a series of continuous cutting paths on the blade surface processed $[17,18]$.

Fig. 3 is a partial enlargement diagram of tool and blade in blade milling process. The residual height is generated by the tool movement along the feed direction and the tool self center rotation. The texture on the surface of milling blade forms due to the above mechanism basically. In addition, when the tool moves along the tool path, the cutting force and the cutting direction of every point on the cutting lane change periodically with the tool rotation. Some phenomenon like the described above enhances the texture of the blade surface, which affects the aerodynamic performance of the blade.

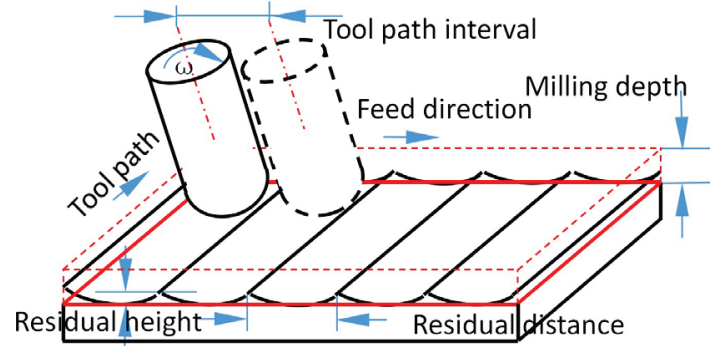

Figure 3 Partial enlargement diagram of tool and blade in blade milling process

Fig. 4(a) shows a NC milled engine blade. We can clearly observe the texture of the blade surface with the naked eye. This texture has been described in the previous discussion, at a magnification of $500 \times$. Under the field of view, Fig. 4(b) is observed. The residual height forms a peak area similar to the shape of a stretch of mountain. To make the reader see the mountain range, the author has marked its position in the micrograph with a dotted red line. Fig. 4(c) more intuitively shows the microscopic topography of the residual height of the milling.
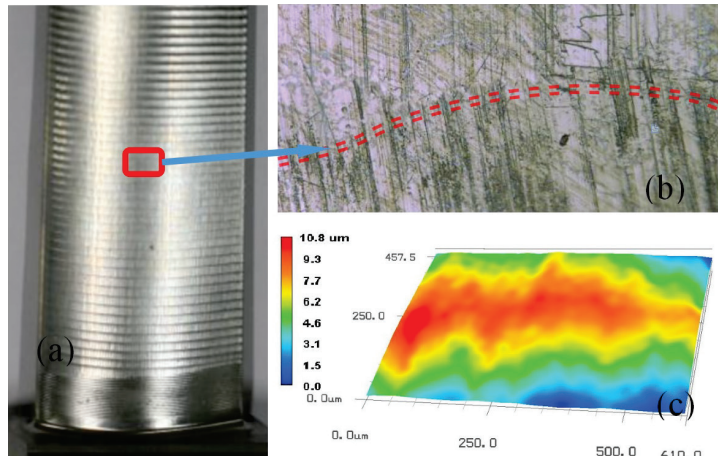

Figure 4 Real-life and magnification map of engine blade by NC milling process

\section{THEORY OF GRINDING ON BLADE SURFACE}

Due to the complex shape surface and the complex relationship between the feed amount and the removal amount, it is difficult to control the amount of material removed. Fig. 5 shows the state of a blade section when it is machined. This figure shows the force relationship between the contact wheel and the blade. $F_{1}$ and $F_{2}$ are the tension forces of the abrasive belt on both sides of the contact wheel, $F_{\mathrm{z}}$ is the support force of the bracket on the contact wheel, $F_{\mathrm{t}}$ is the tangential component of the grinding force, and $F_{\mathrm{n}}$ is the normal component of the grinding force.

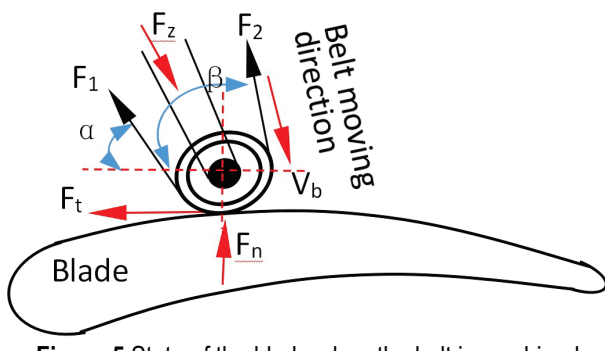

Figure 5 State of the blade when the belt is machined

If the following assumptions are made in the machining process: the contact wheel and the blade are only elastically deformed; the force between them is perpendicular to the contact surface; the geometry of the contact area is a minimum value for the radius of curvature of the contact area; the belt is in a stable grinding process, the above force should satisfy the Eq. (1) relationship. In the contact model shown in figure 6 , the relationship between the force $F_{\mathrm{n}}$ and the indentation displacement amount $D_{\mathrm{p}}$ can be represented by Eq. (2).

$$
\begin{aligned}
& \left\{\begin{array}{c}
F_{1} \cos (\alpha)=F_{t}+F_{2} \cos (\beta) \\
F_{\mathrm{t}}=F_{1}-F_{2}
\end{array}\right. \\
& F_{\mathrm{n}}=\frac{\pi}{4} E^{*} L_{\mathrm{t}} D_{\mathrm{p}}
\end{aligned}
$$

$L_{\mathrm{t}}$ is the contact length and $E^{*}$ is the elastic modulus. The expression of $E^{*}$ is:

$$
\frac{1}{E^{*}}=\frac{1-v_{1}^{2}}{E_{1}}+\frac{1-v_{2}^{2}}{E_{2}}
$$

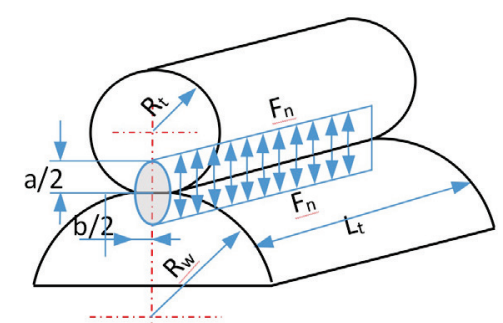

Figure 6 Contact model of blade and contact wheel of abrasive belt system

Among them, $E_{1}, E_{2}$ represent the elastic modulus of the belt contact wheel and blade workpiece, $v_{1}, v_{2}$ represent the Poisson's ratio of the above two. When the radius of the contact section between the blade workpiece and the belt contact wheel is from $a$ to infinity, the contact area can be regarded as a rectangle in the axial direction. The width $2 b$ of the rectangle can be expressed as follows: Description:

$$
\begin{aligned}
& b=\sqrt{R D_{\mathrm{p}}} \\
& \frac{1}{R}=\frac{1}{R_{\mathrm{t}}}+\frac{1}{R_{\mathrm{w}}}
\end{aligned}
$$


where, $R_{\mathrm{t}}$ is the radius of the contact wheel, $R_{\mathrm{w}}$ is the radius of the contact section of the workpiece. When this section is a plane, $R_{\mathrm{w}}$ takes infinite.

In the model in which the contact wheel of the belt contacts the surface of the blade, the concept of the principal curvature $\Sigma \rho$ and the principal curvature function $F(\rho)$ in the Hertzian contact theory can be introduced, namely:

$$
\begin{aligned}
& \sum \rho=\rho_{11}+\rho_{12}+\rho_{13}+\rho_{14} \\
& F(\rho)=\frac{\sqrt{\left(\rho_{11}-\rho_{12}\right)^{2}+2\left(\rho_{11}-\rho_{12}\right)\left(\rho_{21}-\rho_{22}\right) \cos (\omega)+\left(\rho_{21}-\rho_{22}\right)^{2}}}{\sum \rho}
\end{aligned}
$$

In the above equation, $\rho_{11}$ and $\rho_{12}$ represent the two principal curvatures on the two principal planes of the abrasive belt contact wheel at the contact point when it contacts with the workpiece, and similarly, $\rho_{21}$ and $\rho_{22}$ indicate the two principle curvatures of the two principal planes of blade surface, $\omega$ is the angle between the contact wheel and the corresponding principal plane in the vicinity of the contact area of the blade.

The contact problem between the belt contact wheel and the engine blade is more complicated than above description. For analysis of this spatial contact, a small enough analysis area near the contact point is selected, the surface of the area near the contact point is often described as a quadric surface. As shown in Fig. 7, the $x_{1} y_{1}$ plane is the common tangent plane of the area near the contact point. The contact area between the contact wheel and the blade can be represented by the following Eq. (9). There are two points $\mathrm{P}_{1} \mathrm{P}_{2}$ in Fig. $9, \mathrm{P}_{1} \mathrm{P}_{2}$ original distance between them satisfies Eq. (10):

$$
\begin{aligned}
& \left\{\begin{array}{l}
z_{1}=A_{1} x_{1}^{2}+B_{1} y_{1}^{2}+C_{1} x_{1} y_{1} \\
z_{2}=A_{2} x_{1}^{2}+B_{2} y_{1}^{2}+C_{2} x_{1} y_{1}
\end{array}\right. \\
& z_{1}+z_{2}=A_{1} x_{1}^{2}+B_{1} y_{1}^{2}+C_{1} x_{1} y_{1}+A_{2} x_{1}^{2}+B_{2} y_{1}^{2}+C_{2} x_{1} y_{1}= \\
& \quad=\left(A_{1}+A_{2}\right) x_{1}^{2}+\left(B_{1}+B_{2}\right) y_{1}^{2}+\left(C_{1}+C_{2}\right) x_{1} y_{1}
\end{aligned}
$$

If we transform the coordinate system so that the value of $x y$ in the new coordinate system is 0 , according to Galileo invariance, we can get Eq. (11). Under the action of the force, the $\mathrm{P}_{1} \mathrm{P}_{2}$ points produce the displacement of $\lambda_{1} \lambda_{2}$ and come in contact to satisfy Eq. (12). The $\delta$ in the above equation is the elastic approach of the contact wheel and the blade. The pressure expression on the contact area of the contact wheel is Eq. (13).

$$
\begin{aligned}
& z_{1}+z_{2}=A_{0} x^{2}+B_{0} y^{2} \\
& \lambda_{1}+\lambda_{2}=\delta-\left(z_{1}+z_{2}\right)=\delta-\left(A_{0} x^{2}+B_{0} y^{2}\right) \\
& p(x)=p_{\mathrm{m}} \sqrt{1-\left(\frac{x}{a}\right)^{2}} \\
& p(x)=p_{\mathrm{m}} \sqrt{1-\left(\frac{y}{b}\right)^{2}}
\end{aligned}
$$

In the above formula, $p_{\mathrm{m}}$ is the maximum contact pressure, and $p_{\mathrm{m}}=\sqrt{\frac{E^{*} F_{\mathrm{n}}}{\pi L_{\mathrm{t}} R}}$.

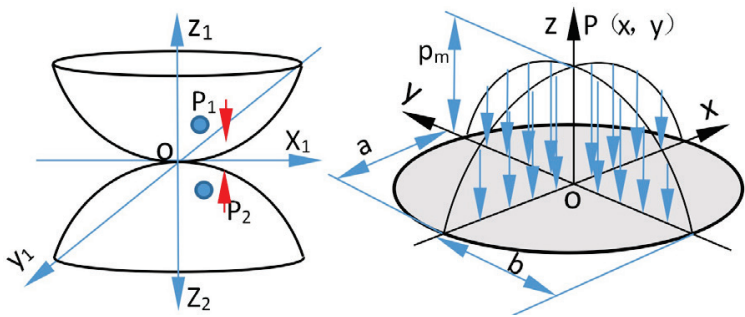

Figure 7 Contact model between two surfaces and the pressure distribution in the contact area

Fig. 7 shows the pressure distribution in the contact area. According to the Hertz contact model, when two surfaces are in close contact and apply a certain pressure, the pressure distribution in the contact area is an ellipsoidal distribution. According to Joseph Valentin Boussinesq's solution, the solution to the pressure distribution in the contact region can be solved. As shown in Fig. 7, $p(x, y)$ is the pressure distribution function in this region, and the contact region is recorded as $\Omega$. The amount of movement along the normal force (that is, the $Z$ direction) at any point in the area can be represented by the integral Eq. (14). In conjunction with Hertz's contact theory, we believe that the contact area is an ellipse as shown in Eq. (15). We can define $p(x, y)$ as Eq. (16):

$$
\begin{aligned}
& z=\frac{1-v^{2}}{\pi E} \iint_{\Omega} \frac{p(\xi, \eta) \mathrm{d} \xi \mathrm{d} \eta}{\sqrt{(x-\xi)^{2}+(y-\eta)^{2}}} \\
& \frac{x^{2}}{a}+\frac{y^{2}}{b}=1 \\
& p(x, y)=p_{0} \sqrt{1-\left(\frac{x}{a}\right)^{2}-\left(\frac{y}{b}\right)^{2}}
\end{aligned}
$$

In above equation, $p_{0}$ is the pressure in the center of the area. In general, $p_{0}=p_{\mathrm{m}}$, this equation is taken into Eq. (14), we can get Eq. (17), in which $K(e)$ is the first type of complete elliptic integral, $D(e)$ and $B(e)$ are the complete combination of the elliptic integrals of the first type and the second type.

$$
\begin{aligned}
& z=\frac{1-v^{2}}{E} p_{0}\left[K(e)-D(e) \frac{x^{2}}{a^{2}}-B(e) \frac{y^{2}}{a^{2}}\right] \\
& K(e)=\int_{0}^{\pi / 2} \frac{1}{\sqrt{\sin (\varphi)}} \mathrm{d} \varphi \\
& D(e)=\frac{1}{e}[K(e)-L(e)] \\
& B(e)=K(e)-D(e) \\
& L(e)=\int_{0}^{\pi / 2} \sqrt{1-e^{2} \sin ^{2}(\varphi)} \mathrm{d} \varphi
\end{aligned}
$$

In above equations, e is the eccentricity of the ellipse $e=\sqrt{1-\left(\frac{b}{a}\right)^{2}}$. Obviously, if $e$ value is determined by 
experiment or by default, the above values can be calculated using the elliptic integral table.

In the stable processing, the total force between the contact wheel and the blade is distributed to the contact area. The area values of the total load and the pressure in the basic area should be equivalent to Eq. (20). Substituting the Eq. (16) of $p(x, y)$ into the Eq. (20), we get the Eq. (21):

$$
\begin{aligned}
& Q=\iint_{\Omega} p(x, y) \mathrm{d} x \mathrm{~d} y \\
& Q=\frac{2 \pi a b}{3 p_{0}}
\end{aligned}
$$

Put any point displacement Eq. (14) mentioned into the contact displacement Eq. (12) at point $\mathrm{P}_{1} \mathrm{P}_{2}$, and decompose $v$ and $E$ into the respective Poisson's ratio $v_{1} v_{2}$ and elastic modulus $E_{1} E_{2}$ of the contact wheel and the blade, we get an implicit integral expression about $p(x, y)$ as Eq. (22), which includes $p(x, y) \Omega$ and $\delta$. If the Hertz description of the contact area is used, then we can substitute Eq. (17) into Eq. (12) to obtain Eq. (23):

$$
\begin{aligned}
& \left(\frac{1-v_{1}^{2}}{\pi E_{1}}+\frac{1-v_{2}^{2}}{\pi E_{2}}\right) \iint_{\Omega} \frac{p(\xi, \eta) \mathrm{d} \xi \mathrm{d} \eta}{\sqrt{(x-\xi)^{2}+(y-\eta)^{2}}}=\delta-\left(A_{0} x^{2}+B_{0} y^{2}\right) \\
& \left(\frac{1-v_{1}^{2}}{\pi E_{1}}+\frac{1-v_{2}^{2}}{\pi E_{2}}\right) p_{0}\left[K(e)-D(e) \frac{x^{2}}{a^{2}}-B(e) \frac{y^{2}}{b^{2}}\right]=\delta-\left(A_{0} x^{2}+B_{0} y^{2}\right)
\end{aligned}
$$

In addition, dealing conjunction with Eq. (21), the following results can be drawn:

$$
\begin{aligned}
& \frac{A_{0}}{B_{0}}=\frac{\left(1-e^{2}\right) D(e)}{B(e)} \\
& a=\varepsilon \sqrt[3]{\frac{3 Q}{2 \sum \rho}\left(\frac{1-v_{1}^{2}}{E_{1}}+\frac{1-v_{2}^{2}}{E_{2}}\right)} \\
& b=\sigma \sqrt[3]{\frac{3 Q}{2 \sum \rho}\left(\frac{1-v_{1}^{2}}{E_{1}}+\frac{1-v_{2}^{2}}{E_{2}}\right)} \\
& \delta=\frac{2 K(e)}{\pi \varepsilon} \sqrt[3]{\frac{1}{8}\left[\frac{3}{2}\left(\frac{1-v_{1}^{2}}{E_{1}}+\frac{1-v_{2}^{2}}{E_{2}}\right)\right]^{2} Q^{2} \sum \rho}
\end{aligned}
$$

In the above equations, $\varepsilon \sigma$ are the coefficients determined by the eccentricity of the ellipse and are generally expressed by the following two equations. It is advisable to rewrite Eq. (11) into Eq. (30), and then substitute Eq. (24) into the Eq. (30) to get Eq. (31).

$$
\begin{aligned}
& \varepsilon=\sqrt[3]{\frac{2 L(e)}{\pi\left(1-e^{2}\right)}} \\
& \sigma=\sqrt[3]{\frac{2 L(e)\left(1-e^{2}\right)}{\pi}} \\
& F(\rho)=\frac{B_{0}-A_{0}}{B_{0}+A_{0}}
\end{aligned}
$$

$$
F(\rho)=\frac{\left[2+\left(1-e^{2}\right)\right] L(e)-2\left(1-e^{2}\right) K(e)}{\left[1-\left(1-e^{2}\right)\right] L(e)}
$$

The point near the contact area of the contact wheel has 0 displacement, that is, $\lambda_{1}=0$. The displacement of the point on the corresponding abrasive belt contact wheel is equal to the elastic approach amount, thus:

$$
\lambda_{2}=\frac{1-v_{2}}{E} b p_{0} K(e)=\delta
$$

In the same process, the curvature of the contact wheel is constant, but the curvature of each section of the blade is inconsistent. This characteristic is particularly obvious at the inlet and exhaust edges.

\section{SIMULATION AND NUMERICAL CALCULATION}

In order to visually show the process characteristics of blade surface grinding by abrasive belts, this paper gives a series of simulations. In some previous studies, many scholars have studied the grinding process of a single abrasive grain on the surface of a titanium alloy, but because the calculation work is too large, the simulation about grinding of the blade surface by the group of abrasives is rarely reported.

The relationship between the contact wheel and the blade surface is shown in Fig. 8(a). Take a piece of micro element in the contact area as the study object. As shown in Fig. 8(b), the abrasive grain in this figure resembles a group of knives with arcs. In this article we regarded them as cutting tools group with a blunt edge. The material on the surface of the blade is deformed under the extrusion action and friction of the abrasive grain group, and then it is turned into chips and finally removed.

The trajectory of the abrasive belt micro-element is shown by the red arrow in Fig. 8(b). This trajectory is the tiny part of the contact belt micro-element contacting the blade surface on the outer contour of the contact wheel. The movement from the start of contact to the contact separation time, the trajectory, the blue arrow indicates that the blade workpiece can be rotated in a certain plane to change the angle between the surface milling texture and the movement path of the belt micro-element.

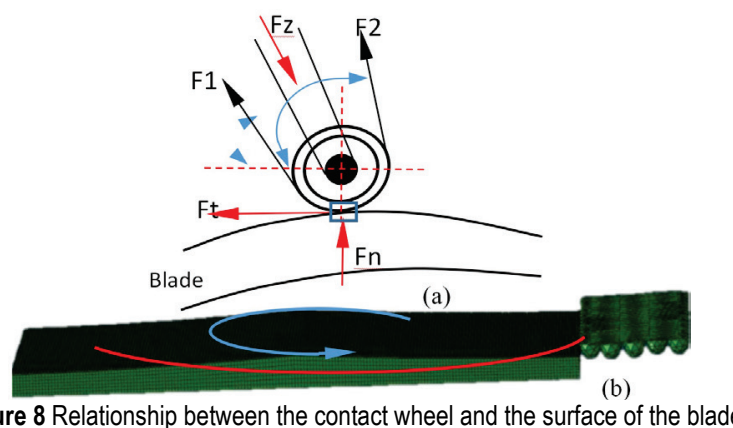

In this paper, the cross-sectional shape of the abrasive grain is regarded as the cross-sectional shape generated by the power-low model. This is because, in many studies, the product surface groove formed by grinding, especially belt grinding, the shape of the abrasive grain generated by the 
power-low model owns the highest similarity. This abrasive grain model cross section can be expressed by the following formula:

$$
W_{\mathrm{g}}=B_{\mathrm{g}} h_{\mathrm{g}}^{E_{\mathrm{g}}}
$$

In the above formula: $E_{\mathrm{g}}$ and $B_{\mathrm{g}}$ are the shape.

The distribution of abrasive grains on the surface of the belt is also an important factor. In general, the penetration depth of the abrasive grains conforms to the Rayleigh Distribution function, and the size of the abrasive grains conforms to the Gaussian distribution function. In order to explore the influence of these factors on the surface morphology and material removal rate of the blade, in this paper, several kinds of belt density model micro elements are selected, andthe abrasive particles are densely distributed on the belt matrix.

The engine blades studied in this paper are compressor blades, whose material is a new titanium alloy named Ti6Al4V. The Johnson-Cook material constitutive model is a thermo-viscoplastic constitutive model suitable for this type of blade material because it is not only suitable for the thin-wall shape characteristics of the blade, but also used for the study of dynamic constitutive relations of titanium alloys, and has a mature numerical calculation program, so that it can often obtain a good effect of grinding mechanism simulation. The mathematical expression of the JohnsonCook material constitutive model is:

$$
\sigma=\left(A+B \varepsilon^{n}\right)\left[1+C\left(\ln \frac{\varepsilon}{\varepsilon_{0}}\right)^{m}\right]\left[1-D\left(\frac{T-T_{0}}{T_{0}}\right)^{k}\right]
$$

In above formula, $A, B, n, C$, and $m$ are material constants, which represent the yield stress strength, strain enhancement coefficient, strain enhancement index, strain rate enhancement parameter, and temperature strain rate sensitivity under quasi-static conditions; $\sigma$ is the flow stress. $T_{\mathrm{m}}$ is the melting temperature; $T_{\mathrm{r}}$ is the reference temperature; $\varepsilon_{0}$ is the reference strain rate. The JohnsonCook material constitutive model parameters of Ti6Al4V are shown in Tab. 1. The grinding process must take into account the failure model parameters of the workpiece material, which are listed in Tab. 2.

Table 1 Johnson-Cook model parameters for Ti6al $4 \mathrm{v}$

\begin{tabular}{|c|c|c|c|c|c|}
\hline$A$ & $B$ & $n$ & $m$ & $T_{\mathrm{m}}$ & $T_{\mathrm{r}}$ \\
\hline 1098 & 1092 & 0.93 & 1.1 & 1630 & 20 \\
\hline
\end{tabular}

Table 2 Parameters for Johnson-Cook model damage

\begin{tabular}{|c|c|c|c|c|c|c|c|}
\hline$d_{1}$ & $d_{2}$ & $d_{3}$ & $d_{4}$ & $d_{5}$ & $T_{\mathrm{m}}$ & $T_{\mathrm{r}}$ & $\begin{array}{c}\text { Reference } \\
\text { strain rate }\end{array}$ \\
\hline-0.09 & 0.25 & 0.5 & 0.014 & 3.87 & 1630 & 20 & 1 \\
\hline
\end{tabular}

In Fig. 9 and Fig. 10 are simulation results of the deformation and removal of the surface micro-elements of the abrasive belt during the movement of the abrasive belt micro-element. It can be seen from Fig. 9 that when the abrasive belt micro-element contacts the blade surface, the deformation area expands rapidly and extends to half of the surface micro-element. The maximum stress occurs at several points where the abrasive particles are in contact with the blade. When the abrasive band micro-element reaches the milling texture peak, the deformation region spreads throughout the blade. As can be seen from Fig. 10, residual stress still exists in the machined micro-element region of the blade surface. When the entire blade surface micro-element is ground, there is still not a large residual stress, as expected before, the original milled texture peak ridge line is not completely removed, and the surface shape at this time is shown in this figure. Fig. 11 shows the abrasive stress and wear on the belt micro-element when the above grinding is completed. The duration of this process is about $0.001 \mathrm{~s}$, and the distance of the belt microelement is $2 \mathrm{~mm}$. Under such circumstances, the belt did not undergo significant wear and damage. By comparison calculation, the material removal thickness at the peak is about 1.93 microns.

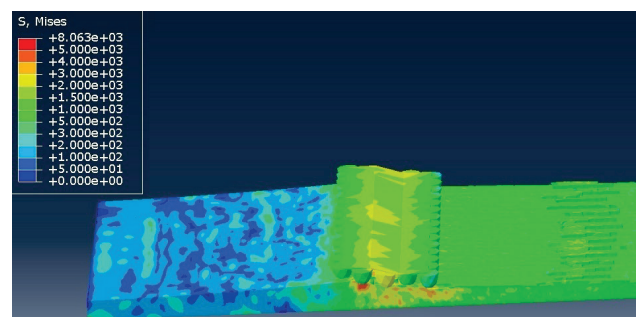

Figure 9 Mises stress at the moment when abrasive belt micro-element reaches the texture peak of blade surface micro-element

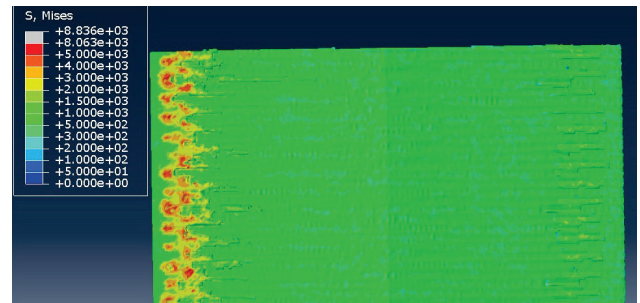

Figure 10 Blade surface shape at the moment when abrasive micro-element leaves from blade micro-element

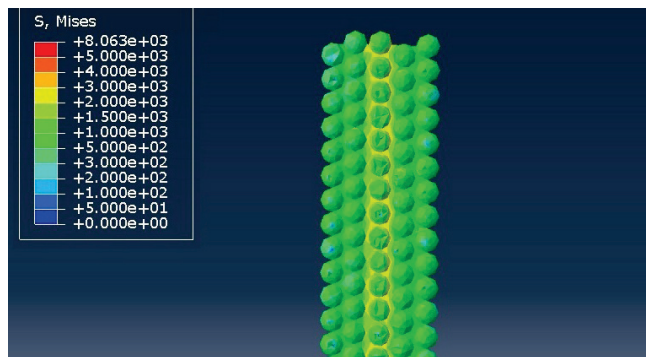

Figure 11 Abrasives stress and wear on the belt micro-element when grinding is completed

In the above process, there must be changes in temperature of the blade surface and the belt abrasives. In order to analyze the temperature change during this process, we read the temperature cloud in the simulation results, as shown in Fig. 12. It is easy to see that the sharp rise in temperature occurs in the area where the chip remains, that is, the valley region of the milled texture. In the peak region of the milled texture, the temperature of the leaf surface micro-element does not rise sharply. This is because: a large amount of heat generated during grinding is attached to the chip body, there is a region where the chip remains, and there is also a phenomenon in which the temperature rises, and there is no chip residue, that is, the chips can be blown away by the cooling high pressure gas. The area does not cause a sharp rise in the surface 
temperature of the blade, so it is very important to remove the chips in time, which can take away a lot of heat.

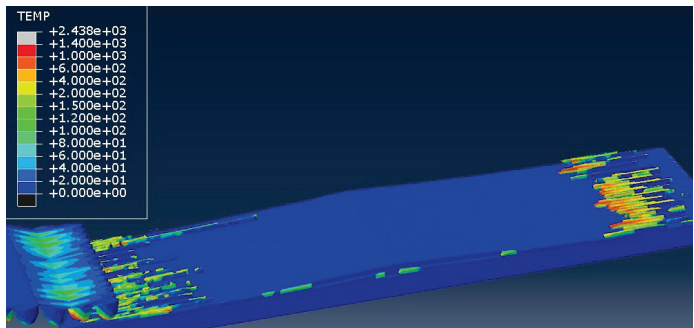

Figure 12 Temperature cloud when abrasive leaves from blade micro-element

We examined the normal plastic strain of the blade surface micro-elements during the micro-machine grinding. Fig. 13 shows the cloud image of this physical quantity. The maximum vertical plastic strain occurs in the milled texture trough. This trough is in the mill. In the first half of the cutting process, as previously analyzed, the contact near the texture peak is unstable, with slip and tremor, then the inherent path of the belt micro-element after it has crossed the milled texture peak will be slightly changed so that the latter half of the grinding action is weakened, and the vertical plastic strain is weakened correspondingly.

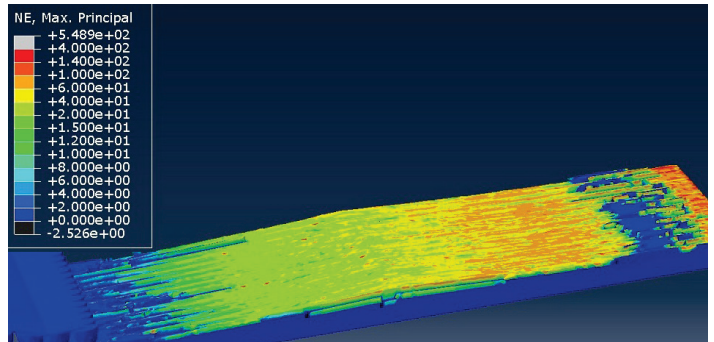

Figure 13 Normal equivalent plastic when abrasive belt micro-element reaches to the texture peak of blade surface micro-element

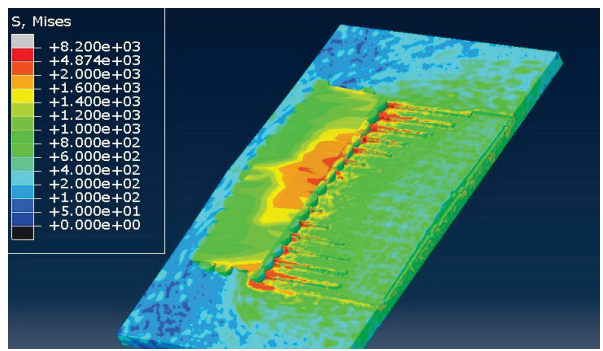

Figure 14 Mises stress when abrasive belt micro-element arrives to the middle line of blade surface micro-element

The angle between the moving direction of the abrasive particle and the peak ridge can be changed. There are changes of various physical quantities when the direction of motion of the abrasive micro-elements along the crest ridge is investigated.

Figs. 14 and 15 show the stress cloud diagram of the grinding process of the abrasive grain micro-element, wherein Fig. 15 is the cloud image and surface topography when the abrasive grain micro-element leaves the blade surface micro-element, in general, in the grinding. In the initial stage, the grinding efficiency is higher, the resulting surface is smoother, and the efficiency is lower at the end of the grinding, with residual chips on the surface of the blade. Similar to the simulation results of the previous group, the single grinding did not remove all the peaks and ridges of the milled texture. This phenomenon can be clearly seen in these figures. The material removal thickness at the peak is about 2.01 microns.

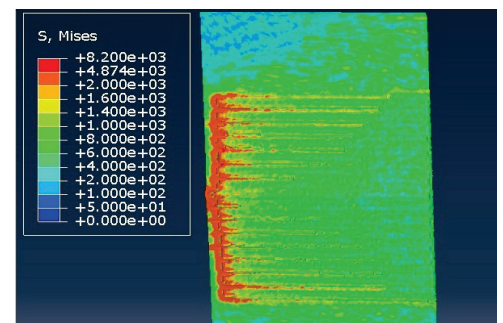

Figure 15 Blade surface shape when abrasive micro-element leaves from blade micro-element

Overall, in the simulation results of this group, the residual stress after grinding is lower than that of the previous group. The values read from the graph are 800 and 1000 , respectively, and the distribution of residual stress is concentrated near the abrasive particles. The change in temperature is also the focus of our attention. As described earlier, the temperature rises sharply because the chips remain on the surface of the blade workpiece, and the heat generated by the grinding cannot be quickly dissipated.

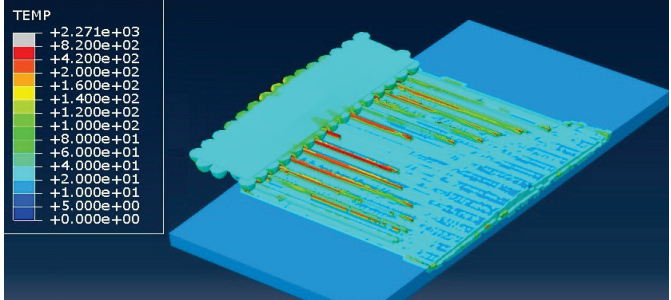

Figure 16 Temperature cloud at the moment when abrasive belt micro-element leaves from blade micro-element

In this group of simulations, although there is no jump disturbance in the milling texture peak, the distribution of the vertical plastic strain still shows similar results to the previous set of simulations, namely: The normal plastic strain in the first half of the grinding is relatively strong, and the vertical plastic strain in the second half is relatively weak. Looking closely at Fig. 17, we can see the residue of the chip interferes with the generation and distribution of vertical plastic strain on the blade surface. Therefore, in the region where the chip does not remain, the vertical plastic strain is strong, and the vertical plastic strain is weak in the region where the chip remains, which is a complementary relationship with the temperature rise law.

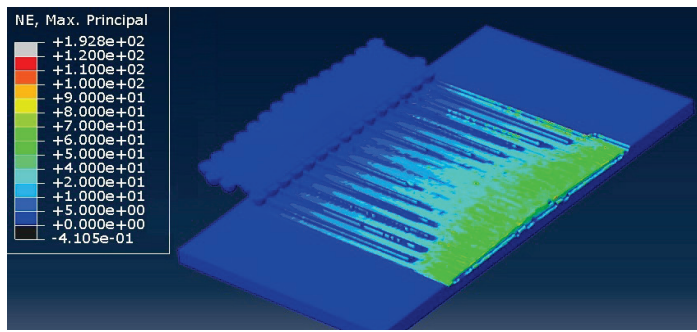

Figure 17 Normal equivalent plastic when abrasive belt micro-element reaches the texture peak of blade surface micro-element

Because the amount of data is too large and the simulation results are complex, we have selected some key data from the simulation results of other groups, and put 
them into a chart form, which is convenient for interpretation and analysis. Figure 18 shows a series of simulation results parameters in different situations of using abrasive belts with different abrasive grain sizes. From the trend of the data curve, in general, the greater the contact force, the more material removed from the blade surface, the greater the local temperature rise of the blade, but this trend is not always maintained. When the contact force is greater than $25 \mathrm{~N}$, the curves tend to be flat. Fig. 19 shows a series of simulation results parameters at different belt running speeds. Similar to the above figure, as the speed of the belt machine increases, the surface material removal and local heating of the blade surface increase. In the comparative analysis of the graph, the increase in the rotational speed is more effective for the removal of the blade material and the local heating.

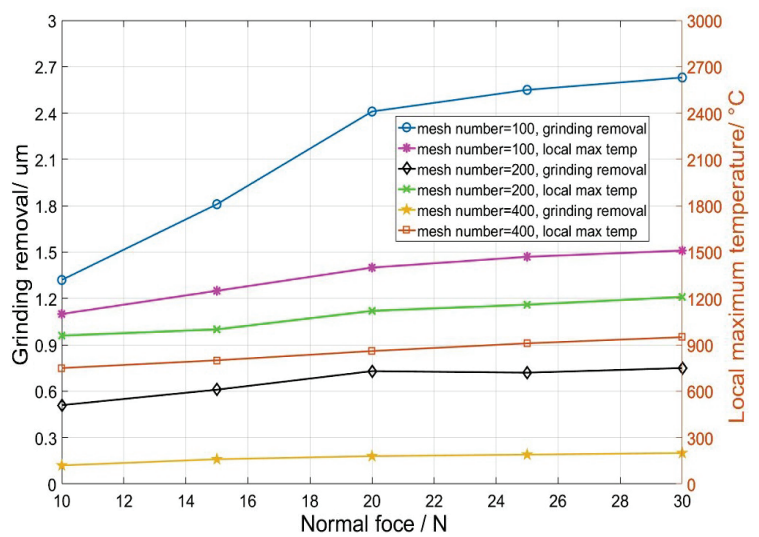

Figure 18 A series of simulation results parameters in different situations of using abrasive belts with different abrasive grains sizes

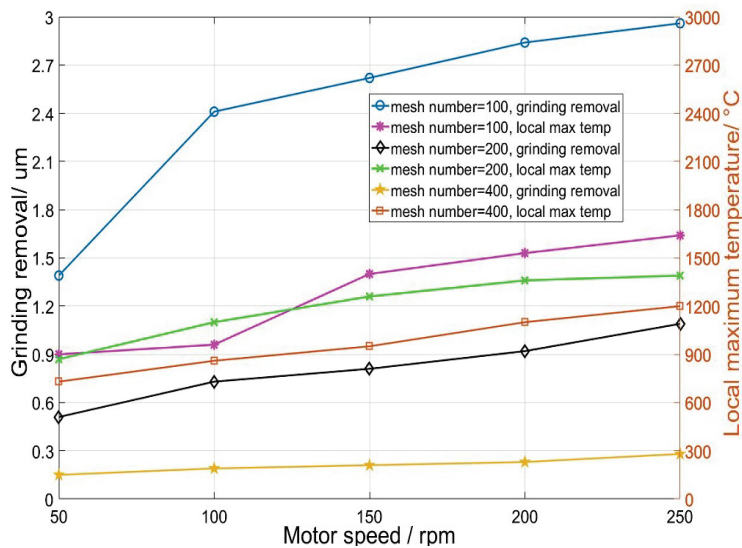

Figure $19 \mathrm{~A}$ series simulation results parameters at different belt running speeds

\section{EXPERIMENT}

Our experiments were carried out on a large 6-DOF robotic system, as shown in Fig. 20. This system contains key components such as robots, belt machines, cooling compressed air, and blade positioners. The robot's manipulator posture can be determined by off-line programming or teaching procedures. The behavior of the manipulator and the movement of the blade positioner work together to make the whole system more complete and suitable for blades with complex three-dimensional shapes.

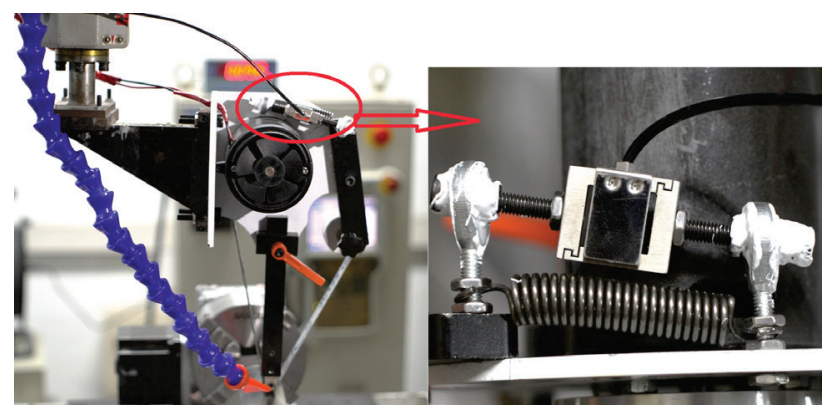

Figure 20 Abrasive belt machine and its control component and application of anti-vibration silica gel to the link joints of the sensor

In order to accurately control the contact force between the belt contact wheel and the blade surface, we installed a high-precision S-type tension and pressure sensor on the belt machine, which is a kind of online monitoring force. The sensor signal is read by the computer acquisition system, yields a series of contact force data that will form real-time feedback from the robot, turning the processing device into a closed-loop-like system that helps improve blade surface accuracy and Processing efficiency. As shown in partial enlargement in Fig. 20, applying antivibration silica gel to the link joints of the sensor can filter out unwanted interference signals, such as the inherent vibration generated by the operation of the belt, which will help improve the system's ability to discriminate between contact forces and reduce data fluctuations. The accuracy of the system. Fig. 21 shows the no-loaded signal from the sensor collected by the computer without anti-vibration silica gel. Fig. 22 shows the no-load signal collected by the computer after the anti-vibration silica gel has been condensed. The comparison between the two shows that the latter signal is more stable and the interference data points are greatly reduced.

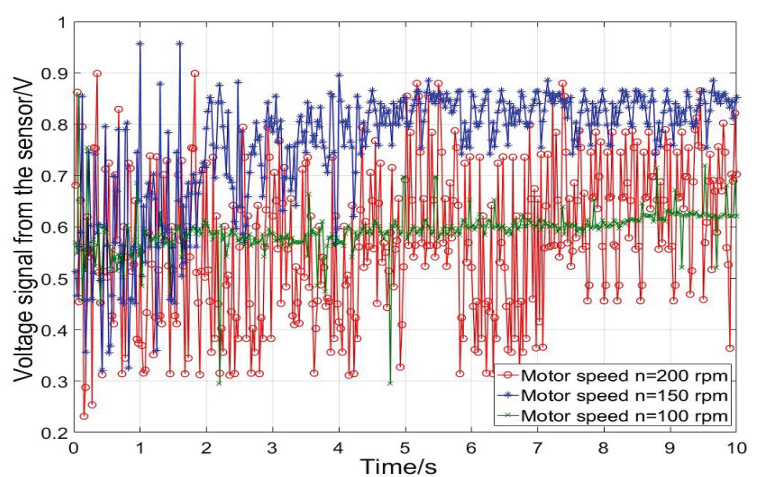

Figure 21 No-loaded signal from the sensor collected by the computer without anti-vibration silica gel

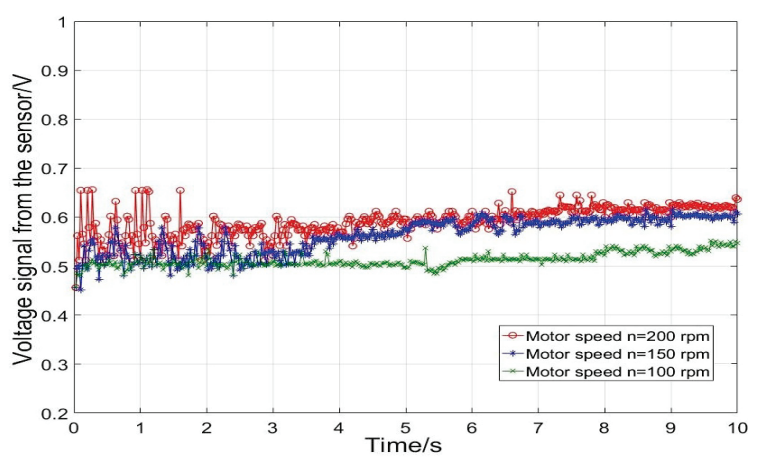

Figure 22 No-load signal collected by the computer after the anti-vibration silica gel has been condensed 
In this processing system, the main control computer is the core component, the robot is the actuator, the rotary platform and the blade positioner are auxiliary parts, and the offline programming software is used to plan the robot arm. The trajectory is determined to form the blade plane to which the belt contact wheel is fitted, and the tension sensor and the data acquisition system form a contact force control system to ensure the uniformity of the force on the surface of the blade. Fig. 23 is a physical diagram of the machining process. The black porous platform can drive the blade to rotate around the z-axis. The servo motor connected to the four-jaw chuck can drive the blade to rotate around the $\mathrm{x}$-axis, so that one has two-dimensional rotation. The functional platform can shorten the adjustment stroke of the robot posture, making it possible for some robots to reach the area, such as the switching of the two surface positions of the blade, the position change of the inlet and exhaust sides of the blade, and the like. Fig. 24 is a close-up photograph of the contact area, the spatial relationship between the belt contact wheel, the blade surface, and the compressed air cooling nozzle can be clearly observed.

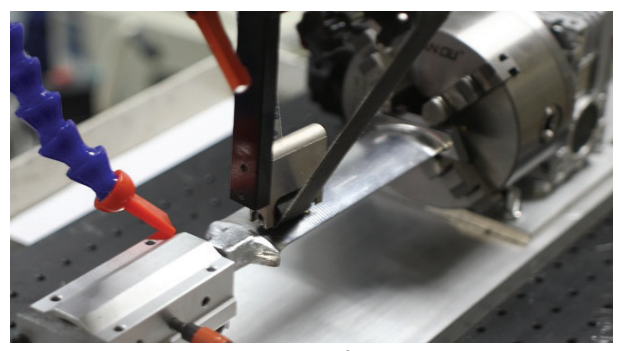

Figure 23 Physical diagram of the machining process

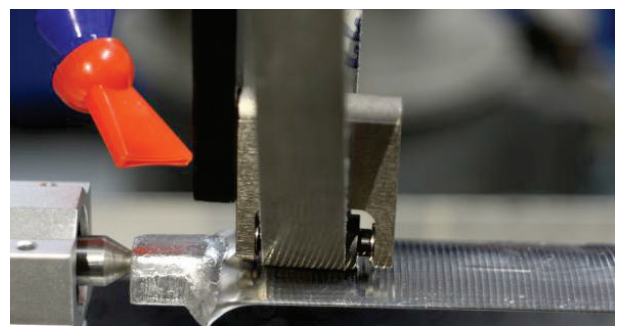

Figure 24 Close-up photograph of the contact area

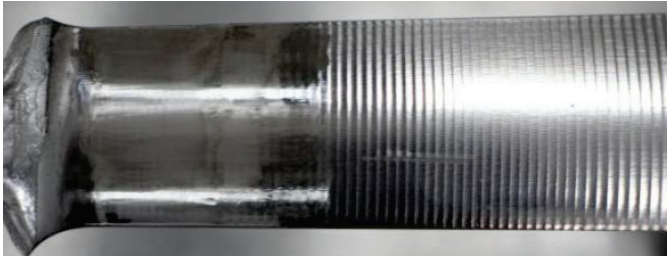

Figure 25 Experimentally obtained back surface of blade

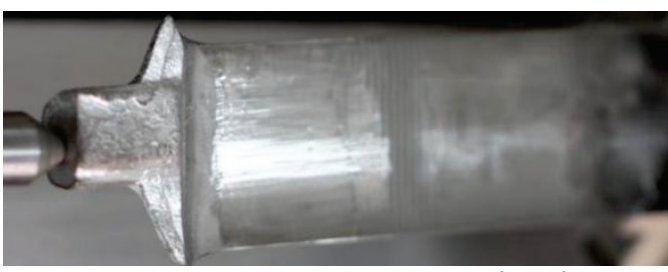

Figure 26 Experimentally obtained basin surface of blade

Fig. 25 and Fig. 26 show experimentally obtained blade surfaces. In order to visually distinguish the experimental results, only the upper half of the blade near the tip of the blade was tested, and the lower surface of the blade root retained the original surface texture. After several times of grinding, the back surface of the blade in Fig. 25 has already exhibited a reflective effect close to the mirror surface. Fig. 26 is the result of grinding the basin surface of the blade. When the number of grinding times and the shape of the contact wheel are kept same, the grinding result of the basin surface will lag behind the back surface.

In order to detect the surface topography error after processing, we selected an area as shown in Fig. 27, in which a square tool setting table was prepared, and these points were measured, and the measured values of these measuring points were compared with the blade design values.

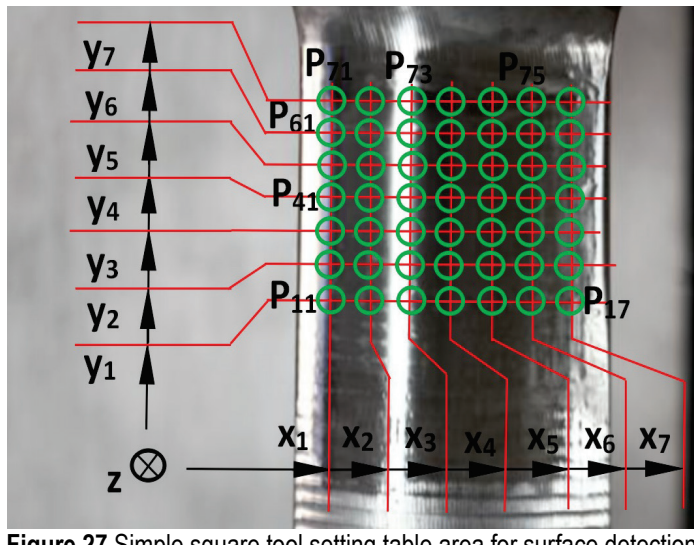

Figure 27 Simple square tool setting table area for surface detection

Tab. 3 shows several values selected from these measurement points, $z_{\mathrm{d}}$ is the design value in the $z$ direction, and $z_{\mathrm{m}}$ is the measurement value in the $z$ direction. It is not difficult to find that the topographical error of the blade surface is controlled within the design error range.

Table 3 Display of measured and design values from selected points on the blade surface

\begin{tabular}{|c|c|c|c|c|c|}
\hline Point & $x / \mathrm{mm}$ & $y / \mathrm{mm}$ & $z_{\mathrm{d}} / \mathrm{mm}$ & $z_{\mathrm{m}} / \mathrm{mm}$ & $z$ error \\
\hline P11 & 11.000 & 10.000 & 15.176 & 15.175 & $\leq 0.02$ \\
\hline P14 & 35.000 & 10.000 & 18.128 & 18.127 & $\leq 0.02$ \\
\hline P22 & 19.000 & 18.000 & 16.375 & 16.362 & $\leq 0.02$ \\
\hline P33 & 27.000 & 26.000 & 17.008 & 16.982 & $\leq 0.02$ \\
\hline P41 & 11.000 & 34.000 & 15.118 & 15.104 & $\leq 0.02$ \\
\hline P65 & 51.000 & 50.000 & 15.808 & 15.781 & $\leq 0.02$ \\
\hline P73 & 27.000 & 58.000 & 15.483 & 15.475 & $\leq 0.02$ \\
\hline
\end{tabular}

Blade surface by design

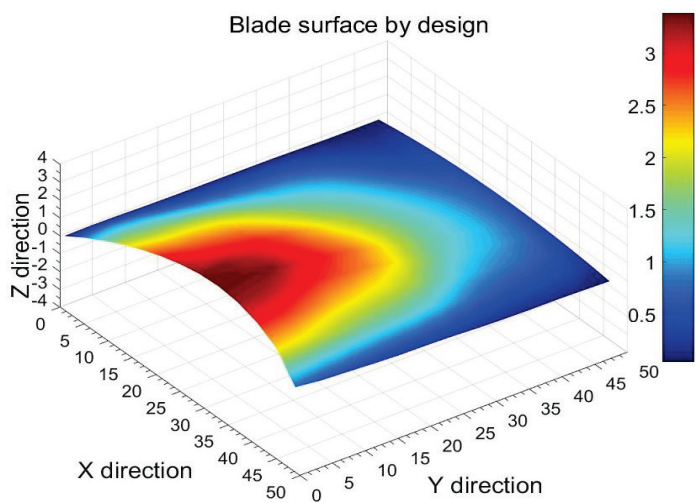

Figure 28 Design morphology of the selected measurement area on the blade surface

In order to visually demonstrate the precision that can be achieved by this method, we draw the design values and 
measured values of the selected points into a threedimensional picture. Fig. 28 shows the design morphology of the selected measurement area on the blade surface. Fig. 29 is a three-dimensional surface drawn by measurement points in the above area. Intuitively, there is no significant difference between the two. The processed surface is very close to the design surface. In other words, the processing experiment is successful.

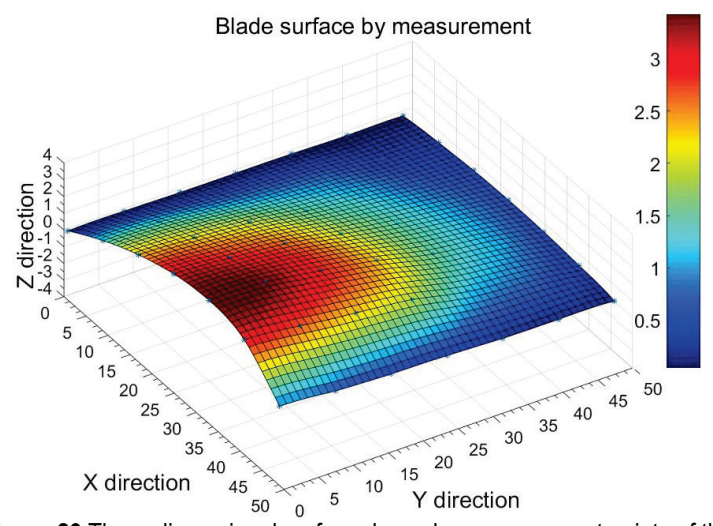

Figure 29 Three-dimensional surface drawn by measurement points of the selected

\section{SUMMARY AND OUTLOOK}

The complexity of the aero-engine and the particularity of its working environment determine that its manufacturing technology must have the highest level of technology. However, in reality, parts with complex shapes such as engine blades still do not reach the level of automatic machining, retaining the original casting and manual grinding process. This paper captures the new trend of the blade production about milling method in NC machining. Based on this, the surface grinding method of the blade oriented to the milling system is proposed. The research in this paper will be beneficial to improve the blade machining. The degree of automation will be so high as to even completely abandon the way of manual grinding. Through a series of studies and experiments, the authors believe that the milled blade, itself and its treatment need to be distinguished from the precision cast blade.

First, the surface texture of the milled blade is significantly different from that of the precision cast surface. The former is a significant discrete surface texture that cannot be simply measured by surface roughness and requires a higher level of measurement. Second, the processing of the surface of the milled blade is more difficult than the precision casting of the blade. This is because during the milling and grinding process, the blade is subjected to different degrees of external force, and the unfavorable factors in the two processes are superimposed. It will make the processing difficulty increase. Third, the milling method will greatly improve the automation of blade processing. At the same time, the working environment of the production workshop will be greatly improved, and even unmanned production will be realized.

What we want to emphasize is that the research in this paper will continue to be carried out in depth, especially in the key areas of path planning of contact wheels, surface fitting of blade surface, improvement of processing quality and processing efficiency. Aero-engine manufacturers all over the world regard these technologies as top secret, and rarely report them. Especially for the research of improving automation, it will be a research hotspot in the field of aeroengine manufacturing technology for a long period of time. Some companies are already at the leading level and we need to work hard to catch up.

\section{REFERENCES}

[1] Koch, C. C. \& Smith, L. H. (1976). Loss Sources and Magnitudes in Axial-Flow Compressors. ASME Journal of Engineering for Power, 98(2), 411-416. https://doi.org/10.1115/1.3446202

[2] Breugelmans, F. A. E. \& Demuth, M. (1984). Influence of Dihedral on the Secondary Flow in a Two-Dimensional Compressor Cascade. ASME Journal of Engineering for Gas Turbines and Power, 106, 578-584. https://doi.org/10.1115/1.3239609

[3] Inoue, M. \& Kuroumaru, M. (1983). Three-Dimensional Structure and Decay of Vortices behind an Axial Flow Rotation Blade Row. ASME Paper 83-GT-69.

[4] Qiu Lei, Zeng Xi, Zi Shiming, et al. (2018). Research on Combustion Chamber Structure Improvement and Ignition System of Missile Turbojet Engine for Diesel Fuel. Tehnicki vjesnik, 25(6), 1792-1800. https://doi.org/10.17559/TV-20180807223114

[5] Smith, L. H. \& Yeh, H. (1963). Sweep and Dihedral Effects in Axial-Flow Turbomachinery. Journal of Basic Engineering, 85, 404-414. https://doi.org/10.1115/1.3656623

[6] Sasaki, T. \& Breugelmans, F. (1998). Comparison of Sweep and Dihedral Effects on Compressor Cascade Performance. Journal of Turbomachinery, 120, 454-464. https://doi.org/10.1115/1.2841738

[7] Zhan, H., Zhao, W., \& Wang, G. (2000). Manufacturing turbine blisks. Aircraft Engineering and Aerospace Technology, 72(3), 247-251. https://doi.org/10.1108/00022660010332032

[8] Berlanger, S., Bordu, S., Maleville, T. J. et al. (2009). Process for manufacturing a single-piece blisk with a temporary blade support ring removed after a milling finishing step: United States, US20110041334A1, 2009-3-25.

[9] Tubbs, H. \& Rea, A. (1991). Aerodynamic Development of the High Pressure Compressor for the IAE V2500 Aeroengine. Imech EC425/023.

[10] Yun, Y. I., Park, I. Y., \& Song, Sj. (2004). Performance Degradation due to Blade Surface Roughness in a Singlestate Axial Turbine. ASME journal of turbomachinery, 127(1), 137. https://doi.org/10.1115/1.1811097

[11] Morita, A., Hattori, S., Tani, K., et al. (1991). Near net shape forging of titantium alloy tuorbineblade. ISLJ International, 31(8), 827-833. https://doi.org/10.2355/isijinternational.31.827

[12] Lu Xian \& Balendra Raj. (2001). Temperature-related errors on aero-foil section of turbine blade. Journal of Materials Pro-cessing Technology, 115(2), 240-244. https://doi.org/10.1016/S0924-0136(01)00817-2

[13] Voigtlander, C. \& Gunther, G. (1983). Isothermal precision forging aero-engine compressor blades made in titanium alloys. Metallurgy, 50(8), 322-326.

[14] Sabau, A. S. \& Visvanathan, S. (2003). Material properties for predicting wax pattern dimensions in investment casting. Material Science and Engineering, A362, 125-134. https://doi.org/10.1016/S0921-5093(03)00569-0

[15] Haihua Wu, Dichen LI, Xiaojie Chen, et al. (2010). Rapid casting of turbine blades with abnormal film cooling holes using integral ceramic casting molds. International Journal of Advanced Manufacturing Technology, 50, 13-19. https://doi.org/10.1007/s00170-009-2502-0

[16] Liu Weiwei, Zhang Dinghua, Shi Yaoyao, et al. (2004). Study on Net-shape NC Machine Technology of Thin blade for 
Aeroengine. Mechanical Science and Technology for Aerospace Engineering, 23(3), 329-331. (in Chinese)

[17] Komanduri, R. (1990). Some Clarifications of the Mechanics of Chip Formation When Machining Titanium Alloys. Journal of Materials Research, 8, 123-125.

[18] Quinsata, Y., Sabourinb, L., \& Lartiguec, C. (2008). Surface topography in ball end milling process: Description of a 3D surface roughness parameter. Journal of Materials Processing Technology, 195, 135-143.

https://doi.org/10.1016/j.jmatprotec.2007.04.129

\section{Contact information:}

\section{Lei QIU}

Zhejiang University of Technology,

Key Laboratory of E\&M, Ministry of Education \& Zhejiang Province

Hangzhou 310032, P. R. China

\section{Xi ZENG}

Zhejiang University of Technology,

Key Laboratory of E\&M, Ministry of Education \& Zhejiang Province

Hangzhou 310032, P. R. China

Shiming J

Corresponding author

Zhejiang University of Technology,

Key Laboratory of E\&M, Ministry of Education \& Zhejiang Province,

Hangzhou 310032, P. R. China

E-mail: jishiming_zjut@163.com

\section{Dapeng TAN}

Key Laboratory of E\&M, Ministry of Education \& Zhejiang Province, Hangzhou 310032, P. R. China

\section{Man GE}

Key Laboratory of E\&M, Ministry of Education \& Zhejiang Province Hangzhou 310032, P. R. China

\section{Wenbin QIU}

Key Laboratory of E\&M, Ministry of Education \& Zhejiang Province, Hangzhou 310032, P. R. China 